\title{
Fast Microwave-Assisted Synthesis of Uniform Magnetic Nanoparticles
}

\author{
Z. Kozakova, P. Bazant, M. Machovsky, V. Babayan and I. Kuritka* \\ Polymer Centre, Faculty of Technology, Tomas Bata University in Zlin \\ Nam. T.G. Masaryka 275, 76272 Zlin, Czech Republic
}

\begin{abstract}
In recent time, magnetic nanoparticles have become widely used for preparation of advanced magnetic materials and also for biomedical applications. Requirement for preparation of particles of suitable shape and size has appeared, hence, various methods have been developed. Here we present rapid and energy saving one-pot solvothermal synthesis using microwave pressurized system. This method allows tuning the size of the particles as well as their magnetic properties. Spherical $\mathrm{Fe}_{3} \mathrm{O}_{4}$ nanoparticles are obtained in 30 min; they are uniform with average dimensions of $200 \mathrm{~nm}$ and exhibit ferromagnetic behavior dependent on synthesis temperature.
\end{abstract}

PACS numbers: 75.50.Tt, 75.60.Ej, 75.75.Cd

\section{Introduction}

In the past decades, the interest in synthesis of uniform magnetic particles in micro- or nanodimensions expanded due to their potential application in many fields. They can be applied in medicine for controlled drug delivery, hyperthermia or medical diagnostic for magnetic resonance imaging. These particles are also used as fillers in magnetic composites or in electromagnetic shielding materials. They are investigated as a key component of magnetorheological fluids, too. Moreover, external magnetic field can be used for preparation of oriented anisotropic structures from this particular system thus achieving novel properties [1-6]. Lots of methods have been introduced for preparation of magnetic particles, employing both dry and wet chemistry. The most popular among them have become simple one-pot solvothermal methods [7-10].

\section{Experimental}

In a standard experiment, $5 \mathrm{mmol}$ of $\mathrm{FeCl}_{3} \cdot 6 \mathrm{H}_{2} \mathrm{O}$ was dissolved in $60 \mathrm{~mL}$ of ethylene glycol, followed by the addition of nucleating agent ( $50 \mathrm{mmol}$ of $\mathrm{NH}_{4} \mathrm{Ac}, 25 \mathrm{mmol}$ of $\left(\mathrm{NH}_{4}\right)_{2} \mathrm{CO}_{3}$ or $200 \mathrm{mmol}$ of aqueous $\left.\mathrm{NH}_{3}\right)$. This mixture was placed in a teflon reaction vessel (XP-1500 Plus), heated in pressurized CEM Mars 5 microwave system (CEM Corporation) to a required temperature $\left(200,210\right.$ or $\left.220^{\circ} \mathrm{C}\right)$ and maintained at this temperature for $30 \mathrm{~min}$. After the reaction, the vessel was cooled to a room temperature and the as-obtained product was filtered off, washed with water and ethanol for

* corresponding author; e-mail: ivo@kuritka.net several times and dried naturally on air. The structure of the final product was characterized by X-ray diffraction method (XRD; PANalytical X'Pert PRO). The particle size and shape was visualized by scanning electron microscopy (SEM; VEGA \\LMU, Tescan) and magnetic properties were identified by a vibrating sample magnetometer (VSM; VSM 7400, Lake Shore).

\section{Results and discussion}

Magnetic particles were obtained by a simple microwave-assisted solvothermal method in $30 \mathrm{~min}$ in nearly $100 \%$. As can be seen from XRD pattern in Fig. 1, all the diffraction peaks are attributed to cubic $\mathrm{Fe}_{3} \mathrm{O}_{4}$, although we cannot distinguish whether it is magnetite or maghemite. Key factor for obtaining well crystallized structures are synthesis time and temperature. The required product can be obtained if the time of synthesis is about $30 \mathrm{~min}$. Experiments with different synthesis temperatures showed that the crystallic structure of particles improves with the temperature increment. The typical SEM image of the product prepared with $\mathrm{NH}_{4} \mathrm{Ac}$ at $220^{\circ} \mathrm{C}$ in Fig. 2 shows that the prepared particles are spherical, uniform and their dimension are about $200 \mathrm{~nm}$. Figure 3 gives magnetization curves of particles prepared with $\mathrm{NH}_{4} \mathrm{Ac}$ at different temperatures and that these particles exhibit ferromagnetic behavior. A strong effect of the synthesis temperature is manifested: with increase of the temperature used for preparation of particles, saturation magnetization and coercivity of prepared material are significantly higher.

\section{Conclusion}

To conclude, in this work there is presented an efficient, rapid and facile one-pot solvothermal microwave-assisted 


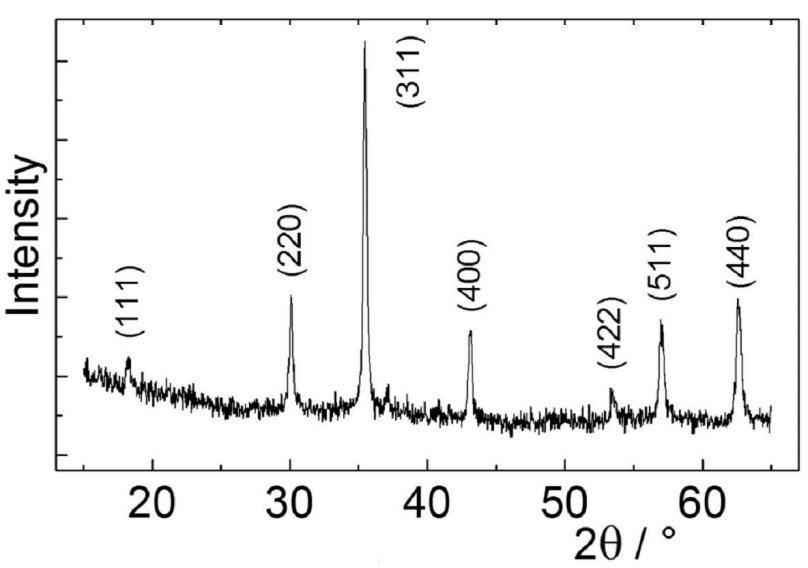

Fig. 1. XRD pattern of $\mathrm{Fe}_{3} \mathrm{O}_{4}$ particles prepared with $\mathrm{NH}_{4} \mathrm{Ac}$ at $220^{\circ} \mathrm{C}$ for $30 \mathrm{~min}$.

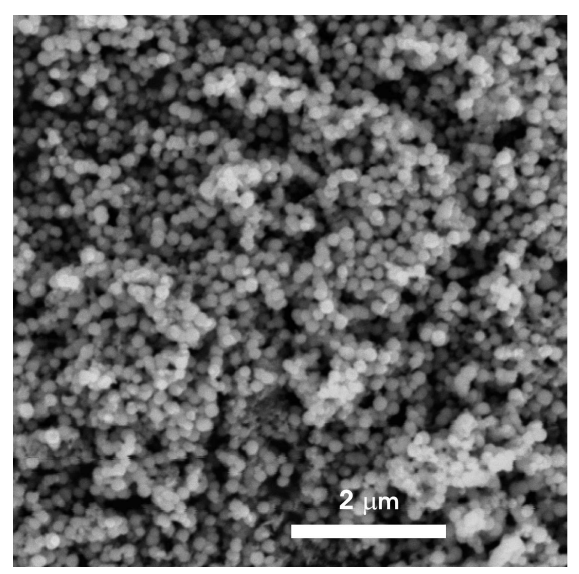

Fig. 2. SEM image of $\mathrm{Fe}_{3} \mathrm{O}_{4}$ particles prepared with $\mathrm{NH}_{4}$ Ac at $220^{\circ} \mathrm{C}$ for $30 \mathrm{~min}$.

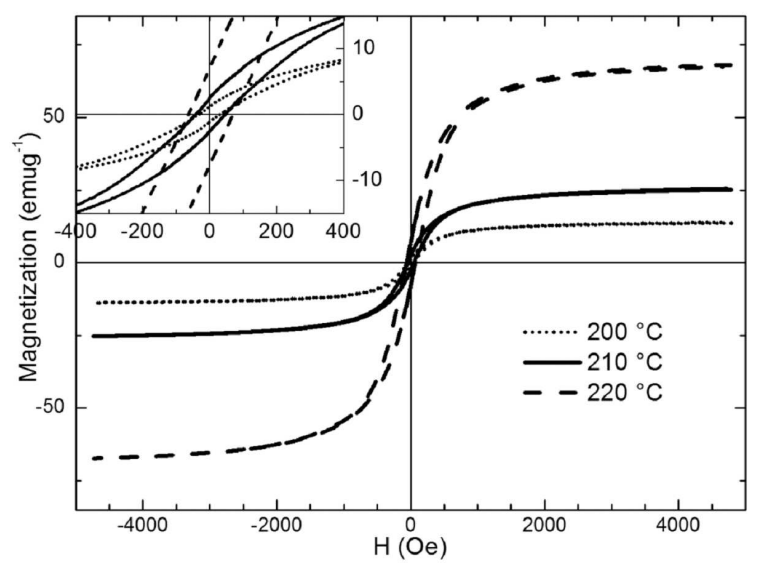

Fig. 3. Room temperature magnetization curves of $\mathrm{Fe}_{3} \mathrm{O}_{4}$ particles prepared with $\mathrm{NH}_{4} \mathrm{Ac}$ at 200,210 and $220^{\circ} \mathrm{C}$ for $30 \mathrm{~min}$. In the inset is given a detailed view of magnetization curves is given, shoving variation of coercivity while different temperatures are used within the synthesis. synthesis of magnetic $\mathrm{Fe}_{3} \mathrm{O}_{4}$ nanoparticles by which they can be obtained in 30 min without requirement of further treatment such as calcination, which can cause disruption of particles. The as-obtained spherical particles are uniform with an average dimension of $200 \mathrm{~nm}$ and exhibit ferromagnetic behavior. The method allows particle size tuning and refinement of magnetic properties by simple changing the synthesis parameters, i.e. temperature or nucleating agent, which can be profitable in future medical application as well as in formation of advanced magnetic materials.

\section{Acknowledgments}

This work was supported by the internal grant of TBU in Zlín No. IGA/25/FT/10/D funded from the resource of specific university research.

\section{References}

[1] A.K. Gupta, M. Gupta, Biomaterials 26, 3995 (2005).

[2] D.K. Kim, Y. Zhang, J. Kehr, T. Kalson, B. Bjelke, M. Muhammed, J. Magn. Magn. Mater. 225, 256 (2001).

[3] A.S. Lübbe, Ch. Alexiou, Ch. Bergemann, J. Surg. Res. 95, 200 (2001).

[4] C. Schao-Wen, Z. Ying-Jie, M. Ming-Yan, L. Liang, Z. Ling, J. Phys. Chem. C 112, 1851 (2008).

[5] J. Jestin, F. Cousin, I. Dubois, C. Ménager, R. Schveins, J. Oberdisse, F. Bouté, Adv. Mater. 20, 2533 (2008).

[6] C. Scherer, A.M. Figueiredo, Neto, Braz. J. Phys. 35, 718 (2005).

[7] H. Peng, Y. Lingjie, Z. Ahui, G. Chenyi, Y. Fangli, J. Phys. Chem. C 113, 900 (2009).

[8] Ch. Xiangying, Z. Zhongjie, L. Xiaoxuan, S. Chengwu, Chem. Phys. Lett. 422, 294 (2006).

[9] Z. Lu-Ping, X. Hong-Mei, Z. Wei-Dong, Y. Guo, F. Shao-Yun, Cryst. Growth Design 8, 957 (2008).

[10] D. Hong, L. Xiaolin, P. Qing, W. Xun, Ch. Jinping, L. Yadong, Angew. Chem. Int. Ed. 44, 2782 (2005). 